\title{
Anthós
}

\section{Toward a Culture of Healing: Why Alternative Therapies and a Feminist Framework are Needed in the Care of Pregnant Women and Treatment of Postpartum Mood Disorders}

Angela Leonardo

Follow this and additional works at: https://pdxscholar.library.pdx.edu/anthos

Part of the Gender and Sexuality Commons, Medicine and Health Commons, and the Women's Studies Commons

Let us know how access to this document benefits you.

\section{Recommended Citation}

Leonardo, Angela (2012) "Toward a Culture of Healing: Why Alternative Therapies and a Feminist Framework are Needed in the Care of Pregnant Women and Treatment of Postpartum Mood Disorders," Anthós: Vol. 4: Iss. 1, Article 3.

https://doi.org/10.15760/anthos.2012.33

This open access Article is distributed under the terms of the Creative Commons Attribution-NonCommercialShareAlike 4.0 International License (CC BY-NC-SA 4.0). All documents in PDXScholar should meet accessibility standards. If we can make this document more accessible to you, contact our team. 
Angela Leonardo

Toward a Culture of Healing: Why Alternative Therapies and a Feminist Framework are Needed in the Care of Pregnant Women and Treatment of Postpartum Mood Disorders

Pregnancy, birth, and early motherhood are areas of human development that have systematically migrated away from their roots as a series of natural life events to a highly, and perhaps unnecessarily, medicalized arena. This shift has been detrimental for women, especially for poor, socially isolated, single, and/or ethnic minority women. In this paper, I outline my concerns with the increased medicalization of birth and postpartum care, as well as with the status of mothers in the United States, and critically examine the patriarchal context in which this shift has occurred. My focus is on maternal health and mortality, including depression and other associated mood disorders during the pre, peri, and postpartum periods. The intent of this paper is not to vilify hospitals or allopathic medicine practitioners, but rather to increase awareness and knowledge of the childbirth process and postpartum period, including procedures and outcomes involved in traditional medical and midwifery care both in and out of the hospital setting. I hope to provide an argument for the use of complementary and alternative therapies and integrative medicine, specifically acupuncture, nutritional supplementation, meditation, and support groups to treat mental health conditions and support overall well-being in pregnant and postpartum women.

I argue that pregnancy and birth are a natural process—one that, in most situations, the body is capable of accomplishing without intervention—but the desire to become a mother and mothering itself may not be as innate as many of us believe. Rather, we are surrounded by the 
“motherhood mystique”, which University of Connecticut Professor of Psychology Mary Crawford describes as "a form of benevolent sexism in which women and men are seen as naturally having different roles and naturally being happy in them” (Crawford, 2006). News correspondent Betty Rollin posits that the "motherhood myth", as she refers to it, proposes as its central tenant "the idea that having babies is something that all normal women instinctively want and need and will enjoy doing” (Rollin, 1970). Crawford (2006) further challenges the premise of the mystique, asking, "If wanting children is instinctive, why are so many powerful socialization forces directed at instilling this 'instinct' in girls?” Children’s playthings, including the market sector dedicated to their production and advertising, illustrates Crawford's (2006) point well. Mattel, a popular U.S. toymaker, manufactures ‘Little Mommy - My Very Real Baby Doll' and markets it to girls as young as two-years-old (Mattel, 2012). The 'Little Mommy' doll includes accessories such as a doll-sized sippy cup, bib, and towel and is billed as a means of promoting nurturing behavior. Mattel's website, which is segregated by gender, does not offer a 'Little Daddy' doll or any other comparable toy for boys of any age that encourages "nurturing playtime” (Mattel, 2012).

Female gender socialization persists well into adulthood and continues to have an impact on grown women's lives. Both the motherhood myth and motherhood mystique present the idea that motherhood is the highest fulfillment for a woman and thus a normal part of the life process, a proposition challenged by some feminist thinkers (Crawford, 2006; Maudro, 2002; Rollin, 1970). If motherhood is in fact a "natural" progression, why then have pregnancy and birth become increasingly medicalized in the U.S. (CDC, 2009; Grady, 2010; Gaskin, 2008; 
Menacker, 2010)? This shift in favor of medicalization is important for feminists to consider because, as Crawford (2006) argues, “Women’s struggle for choice and control in childbirth parallels the struggle for self-determination in general and is part of a social revolution that is not yet complete. The medical model of birth illustrates the way social institutions can decrease the power of women.” When women doubt their competence and self-efficacy and fear the birthing process, they may feel compelled to deliver in a hospital under medical supervision, thus insidiously upholding patriarchal control over women and the female body. Limits on reproductive freedom and choice, including inaccurate, incomplete, or misleading information about reproductive health, are among a contingent of ongoing political and social issues women face in this country, and the debate over the extent of these restrictions extends beyond the wellpublicized contention over birth control pills and abortion and into the delivery room. The choice to have a child, and determine the circumstances around which one desires to birth, is certainly under the purview of reproductive rights.

To be clear, my position is not that there is anything inherently wrong with a hospital birth experience, nor is it that women who birth with the aid of medical intervention are colluding with patriarchy. Birth is a unique experience that varies from woman to woman—and from pregnancy to pregnancy—and many factors can contribute to the need for medical supervision or assistance. Any discourse supporting a preference for natural, un-medicated, vaginal birth as a "real" birthing experience over an anesthetized, hospital, or cesarean birth is divisive and rooted in patriarchal thinking. Cesarean births are indeed real births involving real mothers and babies. Promoting awareness of the many ways women experience birth is 
necessary for uniting women and increases the possibility of facilitating true reproductive justice based on honest dialogue about actual birth experiences. What's more, ignoring improvements in medical treatment that has saved the lives of women and children is irresponsible and dismissive of women's lived experiences. However, such improvements in medicine need to be viewed in the context of an increasing and already high cesarean rate in the U.S. (CDC, 2009; Menacker, 2010; Grady, 2010), and that of a surprisingly high infant and maternal mortality rate compared with other similarly developed countries such as Japan and Norway (Green, 2006; Gaskin, 2008).

Cesarean section has become the most common surgical procedure in American hospitals (Green, 2010), and CDC data from 2007 states that 32\% of births in the U.S. were via C-Section (Menacker, 2010). Dr. Macones, spokesperson for the American College of Obstetrics and Gynecology and chairman of Obstetrics and Gynecology at Washington University in St. Louis, offers this perspective on the rising cesarean rate in the U.S.: "What we're worried about is, the cesarean rate is going up, but we're not improving the health of babies or of moms” (Grady, 2010). This statement could not be truer for African-American women. Veteran midwife Ina May Gaskin (2008) published a compelling article in The Journal of Perinatal Education assessing maternal mortality and the state of maternity care in the U.S. The most striking element of Gaskin’s (2008) article speaks specifically to the rising maternal mortality rate and its notable inequality along racial lines:

“In 1982, the U.S. ratio was 7.5 deaths per 100,000 births. In 2004, it was 13.2 deaths per 100,000. In 2005, the last year for which we have figures, the maternal death ratio was 15.1 deaths per 100,000 births. For African American women, the ratio was an 
outrageous 36.5 deaths per 100,000 births (Kung, Hoyert, Xu, \& Murphy, 2008). In other words, for all U.S. women, the maternal death ratio is almost 5 times as high as it should be, and for African American women, it is more than 10 times what it should be.” Thus, reducing maternal mortality is civil rights issue.

The problem is not simply hospital vs. home birth or cesarean vs. vaginal delivery. Rather, it is that hospitals have become the de-facto choice for pregnant women in this country and that women are not necessarily faring any better as a result. The steady increase in interventions in women's birthing experiences is suggestive of a wider problem with maternal care and the treatment of women, especially in light of grim statistics that show sub-optimal outcomes for women and infants (Gaskin, 2008; Grady, 2010) compared to nations with similar wealth and standards of living (Gaskin, 2008; Green, 2006).

This shift in the U.S. toward increased medical intervention has occurred simultaneously with the proliferation of the concept of the motherhood mystique (Crawford, 2006) and the promotion of the idea that mothering is an instinctive female attribute. Evidence of the mystique is readily apparent in popular media and culture. Entertainment and tabloid magazines and television shows have built a substantial empire showcasing celebrity pregnancies and motherinfant dyads. All parties are perpetually happy, connected, and mutually responsive, and no one appears to be in distress. Sleep deprivation, accurate representations of post-partum bodies, honest depictions of the multitude of birth experiences, and discussion of postpartum depression are largely, and conspicuously, absent in contemporary media. So are positive and affirming representations of mothers of color (Johnston, 2003; Williams, 1994). The day-to-day realities of 
parenting an infant and adjusting to life as a mother are rarely seen or explored in depth, notwithstanding that most of the discussion and depictions of birth in contemporary media are rarely based on any version of reality. The one thing that remains constant is the continued proliferation of a very superficial and narrow account of pregnancy, birth, and mothering and the advancement of the idea that this account is normal.

If motherhood is natural, then postpartum depression is certainly an abomination. There exists a gap between the assumption that mothering is innate, thus requiring little or no support because it is presumably an inborn ability, and the reality of women experiencing a difficult transition into motherhood as indicated by negative or ambivalent reactions to pregnancy and birth, as well as the occurrence of mood disorders in the postpartum period. Despite having one of the most advanced healthcare systems in the world and a high standard of living, it is estimated that eighty percent of women in the United States experience a mild form of depression, dubbed the "baby blues", after giving birth, and that one in five will suffer from postpartum depression (PPD) (Baby Blues, 2005). Symptoms of PPD vary and may include repetitive thoughts, fears or images; irritability; feelings of helplessness or despair; emotional numbness; insomnia or hypersomnia; over-concern for the baby; disinterest in the baby; and frequent sadness or crying (Baby Blues Connection, 2005). The incidence of PPD may actually be higher due to the potential for postpartum conditions to go unreported, untreated, or be misdiagnosed (Baby Blues, 2005). One of the reasons that mood disorders are underreported and underdiagnosed in the U.S. may have to do with the motherhood mystique (Crawford, 2006). Particular aspects of motherhood are celebrated and revered on such a superficial level in 
American society that any outward demonstration or admission of difficulty might be perceived as failing as a mother-and as a woman. If a woman is among the nearly $20 \%$ who experience postpartum depression (Baby Blues, 2005), the stakes are high for her to maintain an illusion of equanimity in a society that concurrently values the idea of females as mothers but provides very little in terms of institutional or social support for new moms. Further, stigmatization of mental illness (Crisp, 2000; Dennis, 2006; Reichler-Rosser, 2003; Sartorius, 2002) in tandem with the motherhood mystique (Crawford, 2006) causes a collective unwillingness to acknowledge that postpartum mood disorders even exist, let alone occur in a significant number of women, resulting in delayed diagnosis and treatment (Dennis, 2006; Reicher-Rossler, 2003) and increased feelings of shame among affected women and their families (Dennis, 2006; ReichlerRossler, 2003). Remarkably, some studies indicate that this stigma is iatrogenic (Dennis, 2006; Sartorius, 2002).

Many lay people and health care practitioners attribute postpartum mood changes to hormonal fluctuations after delivery. However, Crawford (2006) cites compelling research by Treadway et al., (1969) and Johnston-Robledo (2000) that casts serious doubt on this widely-held assumption. Crawford (2006) explains, "Hormone changes have not been shown to cause depression; in fact there is no direct link between postnatal hormone levels and mood.” Instead, a woman's sociocultural context may contribute to how she responds to these hormonal changes: postpartum depression "may be more of a social construction than medical condition. It is virtually unknown in many countries” (Crawford, 2006). Further, Crawford (2006) argues that because of the historically androcentric way in which women's lives are studied, and in which 
women's responsibilities go unnoticed, we may have overlooked a significant and likely cause of postpartum depression—sleep deprivation. Thus, lack of sleep, the demands of caring for an infant, running a household, working, and perhaps dealing with additional stressors brought on by economic insecurity, racism, and lack or absence of familial and social support may have a synergistic negative effect on mental functioning and health, and affect women’s bodies disproportionally and in different ways than men's.

Essentially, we must incorporate an ecological model (Bronfrenbrenner, 1994) that employs a female-centric framework, to successfully understand and assess postnatal adjustment and treat postpartum mood disorders, especially in diverse populations of women. "Early recognition is one of the most difficult challenges with [postpartum depression] because of how covertly it is suffered” (Beck, 2001). Efforts have been made in the nursing community to restructure traditional screens for postpartum depression, in particular the Edinburgh Postnatal Depression Scale, which researchers contend is "not written in the context of new motherhood" (Beck, 2001). A more accurate and precise screen for postpartum depression is the Postpartum Depression Screening Scale (PDSS), developed in 2001 by a nurse-midwife (Beck, 2001). Research indicates that the PDSS differs from traditional postnatal depression screens in its sensitivity (Beck, 2001) — diagnosing maternal depression accurately and early can increase the efficacy of treatment and improve outcomes for mother and child.

Postnatal depression has far-reaching implications; the concern is not only for the mother, but for her child and entire family unit. Extensive studies have documented the effect of maternal depression on infant development (Davis, 2007; McLennan, 2002; Nauert, 2009), but do not 
answer the question of why the U.S. has a significantly higher rate of postpartum mood disorders (Crawford, 2006) and uses medication to treat these conditions more often (Schnyer, 2003; Freeman, 2006, 2009) than other societies around the world. Drawing on Johnston-Robledo's (2000) research, Crawford (2006) offers an important point: “Countries in which postnatal depression is rare offer a period of rest and special care for the new mother, practical and emotional support from other women, and positive attention to the mother, not just the baby.” Further, Crawford (2006) posits that factors like dissatisfaction with postpartum body, feeling overwhelmed and incompetent to care for a newborn, loss of identity in motherhood, and disappointment with partner's lack of support contribute to postpartum depression.

Speaking to this last point, a 2008 Australian study by Bilszta, et al. assessed maternal mental health between single mothers and mothers with negative partner relationships and found that women with unsupportive partners scored higher on tests for depression than single women. Thus, single motherhood alone is not an indicator for potential mental health concerns. Rather, regardless of partner status, all women with pre-existing mood disorders or stressful life events scored worse on depression screens (Bilszta, 2008). The researchers' finding that an unsupportive partner may increase the risk of maternal depression is a noteworthy detail in a patriarchal society that values the sanctity and normalcy of heterosexual marriage. Thus, sociocultural principles and policy promoting the nuclear heterosexual family (Abernathy, 2012; Rochman, 2012) might be misplaced; instead emphasis ought to be on increasing the quality and strength of social ties and support for all women within the broader community. 
Crawford (2006) repeatedly emphasizes the importance of social support for postpartum women and research strongly substantiates this claim. In 2007, Lipman et al. published a study on the "Influence of group cohesion on maternal well-being among participants in a support/education group for single mothers.” This Canadian study validates what single parents and their advocates already know — that receiving social support and other essential resources improves health outcomes for the mother, and in turn the health and well-being of her children. Kaplan et al.'s, 2005 research on the health of impoverished women under welfare reform further highlights the risks of inadequate support. In this study, physiological measurements and diseaserisk markers, such as body mass index (BMI) and blood cholesterol levels, are used to document the increased stress on mothers under "welfare reform" (Kaplan, 2005). Findings reveal that these factors, and the circumstances of women who receive welfare, usually translate to poor or sub-optimal outcomes for mothers and children. Chronic exposure to stressors and inability to reduce stress puts the health of this population at a higher risk for disease than the general population (Kaplan, 2005). If part of the problem is fewer ways to lessen stress, then part of the solution can be to increase the availability of and access to effective stress-reducing programs while simultaneously working to address persistent problems within every level of the sociocultural environment.

Appropriate social support is clearly beneficial and may decrease a woman's risk of developing postpartum depression, as well as associated mood disorders or other negative health outcomes. In addition to Lippman et al.'s (2007) research on the benefits of social support for single mothers, a population especially at risk for social isolation—a factor indicated in 
postpartum mood disorders (Nielsen Forman, 2000) and poor health outcomes_-further studies have documented the importance of strong support networks for community health and wellbeing, even in light of other disease risk factors. Several notable studies came out of Roseto, Pennsylvania between the 1960s-1980s. Roseto, a small manufacturing town comprised almost entirely of Italian immigrants whose men and women were employed in the local slate quarries and blouse mills, respectively, was remarkable for its relatively low incidence of coronary artery disease and heart attack despite a diet rich in animal fat and liberal consumption of alcohol and smoking tobacco, “conventionally accepted risk factors for heart attack” (Bruhn, 1982; Young, 2012). A 1982 article by Bruhn, et al. entitled, “Lessons from Roseto 20 years later: A community study of heart disease” states that, “The major finding was the importance of social support and close family ties in buffering the deleterious effects of stress and life change, factors which have been implicated in the occurrence of myocardial infarction and sudden death.” While the Roseto study did not look specifically at the postpartum population or maternal depression, I believe there is universal applicability in the discovery that social support can not only increase health outcomes, but prevent disease (Young, 2012). This realization is virtually absent from current healthcare policy and social organization; lack of social support is likely contributing to ill health in both the postpartum and general population. In fact, some medical scholars, including Dr. Lauretta Young (2012), retired psychiatrist and professor at Portland State University, and Dr. Mimi Guarneri (2006), cardiologist and author of The Heart Speaks, contend that isolation is a significant factor in disease, citing this timely quote: "The ' $\mathrm{i}$ ' in illness is isolation and the crucial letters in wellness are 'we' (author unknown). 
A suitable complement to support groups and social networks can be found in acupuncture, nutritional supplementation, and mediation. Acupuncture is a form of traditional Chinese medicine (TCM) in which accredited and/or licensed practitioners insert "fine, sterile, single-use surgical steel filaments into the skin to facilitate a redirection and harmonization of the body's vital life force” (Seven Star Acupuncture, 2012). Practiced for centuries in Asia and gaining in popularity and clinical use in the West, acupuncture is concerned with the flow of $q i$, or energy, through the body and has been used to successfully manage and treat conditions such as musculoskeletal pain, insomnia, hypertension, and other manifestations of chronic stress (Farmer, 2012, Grout, 2002). While the exact mechanism by which acupuncture works is unclear, and notwithstanding that the flow or even presence of $q i$ is, at present, difficult to quantify in scientific terms, the positive effects of acupuncture are well documented (Grout, 2002; Hollifield, 2007; Manber, 2004, 2010; Schnyer, 2003). Even the U.S. military has recognized the benefits of traditional Chinese medicine and has trained its doctors to use acupuncture for pain management and in the treatment of post-traumatic stress disorder (PTSD) (Farmer, 2012). In 2003, Schnyer, et al. published an important study assessing the benefits of acupuncture in the treatment of prenatal depression. The researchers recognize that depression during pregnancy is fairly common, especially in the United States. This study highlights the potential negative implications of untreated prenatal depression, and recognizes the difficulty and risks of treating this population with pharmaceuticals. The Schnyer (2003) study concludes that acupuncture is effective, not only in treating, but in immediately relieving some of the physical symptoms of depression. 
Additionally, emerging research in psychiatry is focused on exploring the relationship between diet, nutrients, and mental health. Prolific public health researcher, nutritional epidemiologist, and associate professor at the University of Pittsburg, Lisa Bodnar (2005), published a seminal article on nutrition and maternal mental health, highlighting the fact that, “Adequate nutrition is needed for countless aspects of brain functioning.” Bodnar’s (2005) study suggests that "Poor diet quality...may be a modifiable risk factor for depression" and that "Poor omega-3 fatty acid status increases the risk" of this particular mood disorder. Further studies indicate that deficiencies of several nutrients (e.g. iron, vitamin B12, selenium, essential fatty acids) contribute to or exacerbate mood disorders, including postpartum depression (Beard, 2005; Bodnar, 2005; Corwin, 2003). Bodnar (2005) notes that

“Although nutrient deficiencies are prevalent among all Americans, they disproportionately affect low-income individuals. What is especially intriguing is that socially and economically disadvantaged women are also those at high risk of MDD [Major Depressive Disorder] (Murphy, et al 1991), which suggests that poor nutrition may be an important contributor to mood disorders in this population.”

Research shows that nutritional supplementation, especially omega-3 fatty acids, which are naturally occurring in fish, reduce depressive symptoms and may be helpful in treating depression and mood disorders in postpartum women as stand-alone or adjunctive therapies (Bodnar, 2005; Freeman, 2006, 2009), although some of the researchers indicate that further studies and clinical trials are needed to determine standardized doses (Freeman, 2009). Taken during pregnancy and lactation, essentially fatty-acids appear to have a positive impact on fetal 
and infant neurological development (Parker, 2006). Additionally, Bodnar's (2005) conclusions draw on previous findings indicating that people with depression have deficient levels of folate and vitamin B12 and that supplementing with these nutrients can increase the efficacy of pharmaceutical anti-depressants.

I should note that the use of conventional psychiatric medication is controversial in pregnant and lactating mothers. There are legitimate concerns with using pharmaceuticals in treating depression in this population on molecular, neuroendocrine, and ethical levels. Many women are concerned about the effect of medications on the baby; ceasing breastfeeding to undergo pharmaceutical treatment; and physical separation from the infant in instances of severe depression or psychosis. While the research is divided on the relative risk of certain medications (AHSF, 2012, Davanzo, 2011), some selective serotonin reuptake inhibitors (SSRIs) once considered safe in treating depression in pregnant and breastfeeding mothers, have been linked to medical problems in infants (Davanzo, 2011, Ellfolk, 2010). Prozac, in particular, was associated in one study with a serious lung condition known as persistent pulmonary hypertension of the newborn (PPHN) (AHFS, 2012), but additional studies have not shown a direct link between the drug and PPHN (AHFS, 2012). However, current research urges providers to exercise caution and discretion when prescribing Prozac and other SSRIs in pregnant and lactating women (Davanzo, 2011, Ellfolk, 2010). A significant body of research has indicated that there are medications available that are currently considered safe for use in treating prenatal and postpartum depression (AHFS, 2012, Davanzo, 2011, Ellfolk, 2010). Psychiatrists and other licensed mental health clinicians are trained to help breastfeeding women time their 
feedings when the least amount of medication is in breastmilk. Nonetheless, the question of harm to the infant has not yet been completely eliminated, nor have the long-term health effects of children whose mothers took anti-depressants while pregnant or breastfeeding been adequately studied. This ambiguity and lack of definitive prescribing procedures contributes to women's uneasiness and perhaps unwillingness to seek treatment.

Psychiatric medications have undoubtedly helped many women recover from postpartum mood disorders and reclaim their lives (Baby Blues Connection, 2005). In severe cases such as postpartum psychosis, a medical emergency affecting 1 out of every 1,000 postpartum women (Baby Blues Connection, 2005), treatment with medication is essential. My aim is not to criticize women who chose pharmaceutical treatment for the management of a postpartum mood disorder. In fact, I think it is courageous for a woman to seek out treatment in the midst of the motherhood mystique (Crawford, 2006), especially in a country that does not openly talk about maternal depression. However, we should not ignore the trauma inflicted on thousands of women via unnecessary or excessive treatment with pharmaceuticals, forced hospitalizations, and separation from their children (Baby Blues, 2005). Additionally, a 2006 study by Dennis, et al. assessed barriers to treatment for postpartum depression and, among other conclusions, found that women would prefer "talk therapy" and time and space for discussion of the difficulty with the transition to motherhood from a nonjudgmental provider over conventional treatment with pharmaceuticals.

Less aggressive and more economical treatment options, such as acupuncture, meditation, and diaphragmatic breathing, exist and have been shown to be effective in treating antenatal and 
postpartum depression (Schnyer, 2003; Manber, 2004, Manber, 2010, Vieten, 2008) and in reducing stress (Duesk, 2008; Grout, 2002; Vieten, 2008; Young, 2012). In addition to acupuncture, both meditation and diaphragmatic breathing (as opposed to thoracic breathing) have beneficial and measurable physiological effects on health (Duesk, 2008; Lazar, 2005; Young, 2012). Meditation and diaphragmatic breathing are noteworthy in that they require no interaction with a licensed medical provider, eliminating the issue of cost, nor do they require any specialized equipment, office, or facility.

Complementary and alternative medicine (CAM) is an inherently different, and perhaps anti-patriarchal, way of approaching healthcare, compared to the pathologizing model on which allopathic medicine is based. Integrative and traditional Chinese medicine, including acupuncture, use an ecological model (Bronfrenbrenner, 1994) and must look at the entire picture in order to make an accurate diagnosis and formulate an effective treatment plan. Moreover, unlike allopathic medical care, modalities such as acupuncture treat more than just a specific body part or symptom. Traditional Chinese medicine, and the emerging field of integrative medicine (Young, 2012) emphasize the interconnectedness of body and mind and recognize, much like Kaplan, et al. (2005), Dusek, et al. (2008), Guarneri (2006), and Young (2012), that stress affects the body and can lead to poor health. I submit that the mind-body-spirit connection emphasized in alternative and integrative medicine is inherently feminist in that it provides a subtle challenge to the historical and contemporary culture of objectification, distortion, and dehumanization of women's bodies (Jean Kilbourne, 2000; Ross, 2012). 
Some of the problems with the Western medical model and its treatment of postpartum depression involve the medical deficit model itself. The deficit model of care is focused on a patient's deficiencies, shortcomings, and disease (Valdez, 2010; Young, 2012), and pays little or no mind to her gifts, abilities, or social context. Due to language barriers, poverty, geography, or a combination of these and other factors, poor women and women of color are usually not as able to access alternative treatments or quality medical care, leaving them more susceptible to the negative effects of the medicalization of women's health. The integrative and alternative medicine community, including health care providers who practice or advocate for these modalities, must dedicate more attention to making complementary and alternative care accessible, desirable, and practical for all women. Community acupuncture clinics that offer a sliding scale fee schedule are attempting to tackle this issue (Seven Star Acupuncture, 2012; Working Class Acupuncture, 2008), but their availability varies by region. Acupuncture is just one potent but underused modality for addressing a broad range of uncomplicated yet common conditions affecting women's health. I submit that our society is slowly increasing acceptance of complementary, integrative, and alternative therapies and care, and attention is moving in the direction of promoting positive birth experiences and increased postpartum support for mothers and babies. However, there exist notable disparities in access to complementary and alternative medicine, midwifery, and other non-medicalized care.

Non-Hispanic white, married, and affluent women are using and embracing non-hospital, midwifery and alternative care at a much higher rate than women who are not part of those groups (Baracker, 2011; MacDorman, 2010). I think this shift is somewhat paradoxical 
considering the historical legacy of oppression and racism in hospital admissions policies. Poor women, and especially poor women of color, once had no choice but to deliver their babies unmedicated and at home (Baracker, 2011; Feldhusen, 2000). This has shifted dramatically over the last century; marginalized women now overwhelmingly give birth in a hospital setting (Baracker, 2011; MacDorman, 2010), and women of color, particularly African-American women, have the highest rate of maternal mortality compared to any other racial group in the U.S. (Baracker, 2011; Gaskin, 2008). The existence of this disparity indicates that there is something wrong with the way we care for new mothers. If the "medical model of birth illustrates the way social institutions decrease the power of women” (Crawford, 2006), then let us embrace an alternative that will support all women and recognize their inherent power, not just as mothers, but as individual people. 


\section{References}

Abernethy, S. (2012, March 7). Wisconsin lawmaker introduces law to classify single parenthood as child abuse. Chicagoist. Retrieved from http://chicagoist.com/2012/03/07/wi grothman parenthood abuse.php.

AHFS American Society of Health System Pharmacists, Inc. (2012). AHFS consumer medication information: Fluoxetine. Retrieved from http://www.ncbi.nlm.nih.gov/pubmedhealth/PMH0000885/.

Baby Blues Connection. (2005). Learn More: symptoms, baby blues, postpartum depression.

Retrieved from http://www.babybluesconnection.org/baby blues connection/learn more/symptoms.html

Baracker, L., Dunlap, F. (2011). Disparities in homebirth: The status of homebirth in the U.S.

Whatadoula. Retrieved from http://www.whatadoula.com/blog/blog-lisa.

Beck, C. \& Gabel, R. (2001). Further validation of the postpartum depression screening scale. Nursing Research, 50(3), 155-164. Retrieved from http://journals.lww.com/nursingresearchonline/Abstract/2001/05000/Further Validation of the_Postpartum_Depression.5.aspx.

Bilszta, J., et al. (2008). Single motherhood versus poor partner relationships: Outcomes for 
antenatal mental health. Australian and New England Journal of Psychiatry, 42(1), 56-65.

Retrieved from http://anp.sagepub.com/content/42/1/56.short.

Bodner, L. \& Wisner, K. (2005). Nutrition and depression: Implications for improving mental health among childbearing-aged women. Biological Psychiatry, 58(9), 679-685. Retrieved from http://www.spectracell.com/media/022fullpaper2005biolpsychiatrynutritionanddepression pdf-.pdf.

Bronfrenbrenner, U. (1994). Ecological Models of Human Development. International Encyclopedia of Education, 3. Oxford: Elsiver.

Bruhn, J., et al. (1982). Lessons from Roseto 20 years later: A community study of heart disease. Southern Medical Journal, 75(5), 575-580. Retrieved from http://journals.Iww.com/smajournalonline/Abstract/1982/05000/Lessons_From_Roseto_2 $\underline{0 \text { Years Later A Community.17.aspx. }}$.

CDC - Centers for Disease Control and Prevention. (2009). Press Release March 182009. Retrieved from http://www.cdc.gov/media/pressrel/2009/r090318.htm.

Corwin, E., et al. (2003). Low hemoglobin is a risk factor for postpartum depression. Journal of 
Nutrition, 133(12), 4139-4142. Retrieved from

http://jn.nutrition.org/content/133/12/4139.short.

Crawford, M. (2006). Transformations: Women, gender, and psychology. Boston, MA:

McGrawHill.

Crisp, A., et al. (2000). Stigimatiztion of people with mental illness. The British Journal of Psychiatry, 177, 4-7. Retrieved from http://bjp.rcpsych.org/content/177/1/4.full.

Davanzo, R., et al. (2011). Antidepressant drugs and breastfeeding: a review of the literature [Abstract]. Breastfeeding Medicine, 6(2), 89. Retrieved from http://www.ncbi.nlm.nih.gov/pubmed/20958101.

Davis, E., et al. (2007). Prenatal exposure to maternal depression and cortisol influences infant Temperament [Abstract]. Journal of the American Academy of Child and Adolescent Psychiatry, 46(6), 737-746. Retrieved from http://www.jaacap.com/article/S08908567\%2809\%2962153-5/abstract.

Dennis, C, et al. (2006). Postpartum depression help-seeking barriers and maternal treatment preferences: A qualitative systematic review. Issues in Perinatal Care, 33(4), 323-331. 
Retrieved from: http://onlinelibrary.wiley.com/doi/10.1111/j.1523-

536X.2006.00130.x/abstract?

userIsAuthenticated=false\&deniedAccessCustomisedMessage=

Duesk, J., et al. (2008). Genomic counter-stress changes induced by the relaxation response. PLoS ONE, 3(7). Retrieved from: http://www.plosone.org/article/info\%3Adoi \%2F10.1371\%2Fjournal.pone.0002576.

Ellfolk, M. \& Malm, H. (2010). Risks associated with in utero and lactation exposure selective serotonin reuptake inhibitors (SSRIs) [Abstract]. Reproductive Toxicology, 30(2), 249260. Retrieved from http://www.sciencedirect.com/science/article/pii/S0890623810000936.

Farmer, B. (2012). Military pokes holes in acupuncture skeptics’ theory. NPR. Retrieved from http://www.npr.org/2012/02/16/146944270/military-pokes-holes-inacupuncture-skeptics-theory.

Feldhusen, A. (2000). The history of midwifery and childbirth in America: A timeline. Midwifery Today. Retrieved from http://www.havingababytoday.com/articles/timeline.asp.

Freeman, M. (2006). Omega-3 fatty acids and perinatal depression: A review of the literature and 
recommendations for future research [Abstract]. Prostaglandins, Leukotrienes and Essential Fatty Acids, 75(4-5), 291-297. Retrieved from http://www.sciencedirect.com/science/article/pii/S0952327806001219.

Freeman, M. (2009). Complementary and alternative medicine for perinatal depression. Journal of Affective Disorders, 112, 1-10. http://www.sciencedirect.com/science/article/pii/S0165032708002747.

Gaskin, I. (2008). Maternal death in the United States: A problem solved or a problem ignored?. Journal of Perinatal Education, 17(2), 9-13.

Grady, D. (2010). Cesarean births are at a high in the US. The New York Times. Retrieved from http://www.nytimes.com/2010/03/24/health/24birth.html.

Green, J. (2006). US has second worst newborn death rate in modern world, report says. CNN. Retrieved from http://articles.cnn.com/2006-05-08/health/mothers.index_1_mortalityrate-death-rate-world-s-mothers? s=PM:HEALTH.

Grout, M. (2002). Medical acupuncture in the treatment of chronic stress related illness. Acupuncture Today, 3(8). Retrieved from http://www.acupuncturetoday.com/archives2002/aug/08grout.html

Guarneri, M. (2006). The heart speaks: A cardiologist reveals the secret language of healing. 
New York, NY : Simon \& Shuster.

Hollifield, M., et al. (2007). Acupuncture for posttraumatic stress disorder: A randomized controlled pilot trial. Journal of Nervous and Mental Disease, 195(6), 504-513.

Retrieved from

http://www.northeastintegrativemedicine.org/resources/ResearchReferences/Acupuncture /PosttraumaticStressDisorder.pdf.

Jean Kilbourne (Producer). (2000). Killing us softly 3 [Motion Picture]. Northampton, MA: Media Education Foundation.

Johnston, D. \& Swanson, D. (2003). Invisible mothers: A content analysis of motherhood ideologies and myths in magazines. Sex Roles, 49. Retrieved from http://www.public.asu.edu/ kleong/mothers\%20in\%20magazines.pdf.

Kaplan, G., et al. (2005). The health of poor women under welfare reform. American Journal of Public Health, 95(7), 1252-1258.

Lazar, S., et al. (2005). Meditation experience is associated with increased corticol thickness. Neuroreport, 16(17), 1893-1897.

Lipman, E., et al. (2007). Influence of group cohesion on maternal well-being among participants in a support/education group program for single mothers. American Journal of Orthopsychiatry, 77(4), 543-549. 
Manber, R., et al. (2004). Acupuncture: A promising treatment for depression during pregnancy. Journal of Affective Disorders, 83(1), 89-95. Retrieved from http://www.sciencedirect.com/science/article/pii/S0165032704001879.

Manber, R., et al. (2010). Acupuncture for depression during pregnancy: A randomized controlled trial. Obstetrics and Gynecology, 115(3), 511-520. Retrieved from http://journals.lww.com/greenjournal/Fulltext/2010/03000/Acupuncture for Depression During Pregnancy A.7.aspx?WT.mc id=HPxADx20100319xMP.

MacDorman, et al. (2010). Trends and characterizes of home and other out of hospital births in the United States, 1990-2006. National Vital Statistics Report, 58(11), 1-15. Retrieved from http://www.cdc.gov/nchs/data/nvsr/nvsr58/nvsr58_11.pdf.

McLennan, J. \& Offord, D. (2002). Should postpartum depression be targeted to improve child mental health?. Journal of the American Academy of Child and Adolescent Psychiatry, 41(1), 28-35. Retrieved from http://www.sciencedirect.com/science/article/pii/S0890856709605852.

Menacker, F. \& Hamilton, B. (2010). Recent trends in cesarean delivery in the United States. NCHS data brief, 35. National Center for Health Statistics: Hyattsville, MD. Retrieved from http://www.cdc.gov/nchs/data/databriefs/db35.pdf. 
Mattel, Inc. (2012). Little Mommy Scrub A Dub Doll. Retrieved from http://shop.mattel.com/product/index.jsp?productId=12501577.

Maudro, E. (2002). Excuse me while I explode: My mother, myself, and my anger. In Peterson, L., Brereton, J. (Eds.). The Norton Reader. (pp. 255-261). New York, NY: Norton.

Nauert, R. (2009). Postpartum depression's effect on the baby. Psych Central.

Retrieved from http://psychcentral.com/news/2009/08/21/postpartum-depressions-effecton-the-baby/7899.html.

Nielsen Forman, D., et al. (2000). Postpartum depression: Identification of women at risk. BJOG: An International Journal of Obstetrics and Gynecology, 107(10), 1210-1217. Retrieved from http://onlinelibrary.wiley.com/doi/10.1111/j.14710528.2000.tb11609.x/full.

Parker, G., et al. (2006). Omega-3 fatty acids and mood disorders. American Journal of Psychiatry, 163(6). Retrieved from http://www.pn.psychiatryonline.org/article.aspx? articleID $=96663 \& a t a b=7$.

Reicher-Rossler, A., et al. (2003). Postpartum depression: do we still need this diagnostic term? Acta Psychiatrica Scandinavica, 108, 51-56. Retrieved from http://onlinelibrary.wiley.com/doi/10.1034/j.1600- 
0447.108.s418.11.x/abstract;jsessionid=2BF30F3B6BF81B955FCB5FAB483E1CB4.d01 t01 ?userIsAuthenticated $=$ false $\&$ deniedAccessCustomisedMessage $=$.

Rochman, B. (2012, March 14). According to a Wisconsin bill, single moms are a child abuse threat. Time. Retrieved from http://healthland.time.com/2012/03/14/according-to-a-wisconsin-bill-single-moms-are-achild-abuse-threat/.

Rollin, B. (1970). Motherhood: Who needs it? In Peterson, L., Brereton, J. (Eds.). The Norton Reader. (pp.369-377). New York, NY: Norton.

Ross, J. (2012, April). Feminist pedagogy and feminist science studies: Teaching inclusive science. Lecture conducted for the Northwest Women’s Studies Association Conference at Portland State University. Portland, Oregon.

Sartorious, N. (2002). Iatrogenic stigma of mental illness. British Medical Journal, 324(7352), 1470-1471. Retrieved from http://www.ncbi.nlm.nih.gov/pmc/articles/PMC1123430/.

Schnyer, R., et al. (2003). Acupuncture treatment for depression during pregnancy: Conceptual: framework and two case reports. Complementary Health Practice Review, 8(1), 40-53. Seven Star Acupuncture. (2012). Services: Acupuncture. Retrieved from http://sevenstarpdx.com/. 
Valdez, M. (2012, April). Lecture conducted for Psychology 310 Psychology of Women at Portland State University. Portland, Oregon.

Vieten, C. \& Astin, J. (2008). Effects of a mindfulness-based intervention during pregnancy on pre-natal stress and mood: Results of a pilot study. Archives of Women's Mental Health, 11(1), 67-74. Retrieved from http://resources.metapress.com/pdf-preview.axd? code $=$ u718t0802658447g\&size=largest.

Young, L. (2012, April). Lecture conducted for Public Health Education 275 Stress Management at Portland State University. Portland, Oregon.

Williams, L. (1994). Race, rat bites and unfit mothers: How media discourse informs welfare legislation debate. Fordham Urban Law Journal, 22(4). Retrieved from http://ir.lawnet.fordham.edu/cgi/viewcontent.cgi?article=1440\&context=ulj.

Working Class Acupuncture. (2008). Retrieved from http://www.workingclassacupuncture.org/. 